\title{
THE REPRESENTATION OF REAL NUMBERS
}

\section{O. W. RECHARD}

1.0. Introduction. Let $p \geqq 2$ be a positive integer and denote by $E_{p}$ the class of all continuous, strictly increasing functions $f(x)$ on the interval $0 \leqq x \leqq p$ with $f(0)=0$ and $f(p)=1$. In a generalization of the decimal representation, Everett ${ }^{1}$ has associated with every real number $0 \leqq \gamma<1$ a sequence of integers $c_{1}, c_{2}, \cdots$, with $0 \leqq c_{i} \leqq p-1$ by means of the following algorithm:

$$
\begin{array}{ll}
\gamma=f\left(\gamma_{1}\right), & 0 \leqq \gamma_{1}<p, \\
\gamma_{1}=c_{1}+f\left(\gamma_{2}\right), & 0 \leqq \gamma_{2}<p, \\
\gamma_{2}=c_{2}+f\left(\gamma_{3}\right), & 0 \leqq \gamma_{3}<p,
\end{array}
$$

where $f(x)$ is an arbitrary function in the class $E_{p}$.

Some functions (for example, $f(x)=x / p$, which leads to the representation of a number as a decimal to the base $p$ ) when employed in the algorithm (A) yield one-one correspondences between real numbers and sequences of integers mod $p$. On the other hand, any function, for example, whose graph has more than one point in common with any of the straight line segments connecting the points $(j, 0)$ and $(j+1,1), j=0,1, \cdots, p-1$, will obviously lead to a correspondence which is many-one. We shall denote by $E_{p}^{*}$ the subclass of $E_{p}$ consisting of those functions which in the algorithm (A) give rise to one-one correspondences.

The present paper contains very simple characterizations of those correspondences between real numbers and sequences of integers $\bmod p$ which can be obtained by applying the algorithm (A) with functions from the classes $E_{p}^{*}$ and $E_{p}-E_{p}^{*}$ respectively. By means of these characterizations it is possible to settle two of the problems raised by Everett and to give an answer (albeit not a completely satisfactory one) to a third, namely that of characterizing the class $E_{p}^{*}$ itself.

2.0. Associated functions. For every function $f(x)$ in $E_{p}$ we can define an associated function $F(x)$ on $0 \leqq x \leqq 1$ as follows. Define $F(1)=1$. If $0 \leqq \gamma<1$, let $c_{1}, c_{2}, \cdots$ be the sequence of integers asso-

Presented to the Society, November 26, 1948; received by the editors August 12 , 1949.

${ }^{1}$ Representations for real numbers, Bull. Amer. Math. Soc. vol. 52 (1946) pp. 861869. 
ciated with $\gamma$ by the algorithm (A) utilizing the function $f(x)$; then define $F(\gamma)=\sum_{j=1}^{\infty} c_{j} / p^{j}$.

2.1. TheOREM 1. If $f(x)$ is any function in the class $E_{p}$, its associated function $F(x)$ is continuous and nondecreasing on $0 \leqq x \leqq 1$.

Proof. Note first that for $0 \leqq \gamma<1, F(\gamma) \leqq \sum_{j=1}^{\infty} p-1 / p^{i}=1=F(1)$. Now fix any two points $\alpha$ and $\beta$ such that $0 \leqq \alpha<\beta<1$, and let the sequences obtained from applying (A) to $\alpha$ and $\beta$ be respectively $a_{1}, a_{2}, \cdots$, and $b_{1}, b_{2}, \cdots$. If $F(\alpha) \neq F(\beta)$, let $n$ be the first integer such that $a_{n} \neq b_{n}$. At the $(n+1)$ st stage in applying (A) to $\alpha$ and $\beta$ we have respectively

$$
\begin{aligned}
& \alpha_{n}=a_{n}+f\left(\alpha_{n+1}\right), \\
& \beta_{n}=b_{n}+f\left(\beta_{n+1}\right),
\end{aligned} \quad f\left(\alpha_{n+1}\right), f\left(\beta_{n+1}\right)<1,
$$

and since the monotonicity of $f(x)$ implies $\alpha_{n}<\beta_{n}$ it follows that $a_{n}<b_{n}$. Consequently, $F(\alpha)<F(\beta)$, and $F(x)$ is nondecreasing on $0 \leqq x \leqq 1$.

Clearly, the range of values of $F(x)$ includes every terminating decimal on $[0,1]$. In fact, if $c_{1}, c_{2}, \cdots, c_{n}$ is any finite sequence of integers $\bmod p$, then

$$
F\left[f\left(c_{1}+f\left(c_{2}+\cdots+f\left(c_{n}\right) \cdots\right)\right)\right]=0 . c_{1} c_{2} \cdots c_{n} .
$$

Consequently, since the terminating decimals are dense on $[0,1]$ and $F(x)$ is monotonic, it follows that $F(x)$ is continuous and, in fact, takes on every value on $[0,1]$.

2.2. Note that for $f(x) \in E_{p}$ its associated function is uniquely defined by the functional equation

$$
F[f(x)]=[x] / p+F(x-[x]) / p, \quad 0 \leqq x \leqq p,
$$

where $[x]$ denotes the largest integer in $x$. Certainly, the associated function for $f(x)$ satisfies (B). Assume that a function $F(x)$ defined on $[0,1]$ satisfies $(B)$; then $F(0)=0$ and $F(1)=1$. Moreover, if under the algorithm (A), $\gamma<1$ corresponds to the sequence $c_{1}, c_{2}, \cdots$, then we have

$$
\begin{array}{ll}
\gamma=f\left(\gamma_{1}\right), & 0 \leqq \gamma_{1}<p, \\
\gamma_{1}=c_{1}+f\left(\gamma_{2}\right), & 0 \leqq \gamma_{2}<p, \\
\gamma_{2}=c_{2}+f\left(\gamma_{3}\right), & 0 \leqq \gamma_{3}<p,
\end{array}
$$

and 


$$
\begin{aligned}
F(\gamma) & =F\left[f\left(\gamma_{1}\right)\right]=c_{1} / p+F\left[f\left(\gamma_{2}\right)\right] / p \\
& =c_{1} / p+c_{2} / p^{2}+F\left[f\left(\gamma_{3}\right)\right] / p^{2}=\cdots .
\end{aligned}
$$

2.3. If $F\left(x_{1}\right)=F\left(x_{2}\right)=c$ for $x_{1}<x_{2}$ (and, hence, $F(x)=c$ for $x_{1} \leqq x$ $\leqq x_{2}$ ), then $c$ is called a level value for $F(x)$. It is easy to see that $F(x)$ has the following property: if the algorithm (A) when applied to two distinct numbers $\alpha$ and $\bar{\alpha}$ yields in each case the sequence $a_{1}, a_{2}, \cdots$, so that $0 . a_{1} a_{2} \cdots$ is a level value for $F(x)$, then each of the numbers $0 . a_{2} a_{3} \cdots$ and $0 . i a_{1} a_{2} \cdots, i=0,1, \cdots, p-1$, must also be a level value. For if

$$
\begin{array}{ll}
\alpha=f\left(\alpha_{1}\right), & 0 \leqq \alpha_{1}<p, \\
\alpha_{1}=a_{1}+f\left(\alpha_{2}\right), & 0 \leqq \alpha_{2}<p,
\end{array}
$$

and

$$
\begin{array}{ll}
\bar{\alpha}=\quad f\left(\bar{\alpha}_{1}\right), & 0 \leqq \bar{\alpha}_{1}<p, \\
\bar{\alpha}_{1}=a_{1}+f\left(\bar{\alpha}_{2}\right), & 0 \leqq \bar{\alpha}_{2}<p,
\end{array}
$$

then the two distinct numbers $\alpha_{1}-a_{1}$ and $\bar{\alpha}_{1}-a_{1}$ each yield under (A) the sequence $a_{2}, a_{3}, \cdots$. Similarly, the two numbers $f(\alpha+i)$ and $f(\bar{\alpha}+i)$ each yield the sequence $i, a_{1}, a_{2}, \cdots$.

This property of the function $F(x)$ implies that it is either strictly increasing (as is the case if and only if $f(x)$ is in $E_{p}^{*}$ ) or else its level values, and hence its intervals of constancy, are dense on $[0,1]$.

3.0. The one-one case. If $f(x) \in E_{p}^{*}$, its associated function is continuous and strictly increasing on $[0,1]$ to $[0,1]$. We next inquire whether or not every such function is associated with some function in $E_{p}^{*}$.

THEOREM 2. If $F(x)$ is any continuous, strictly increasing function on $0 \leqq x \leqq 1$ with $F(0)=0$ and $F(1)=1$, then it is the associated function for a unique function $f(x)$ in the class $E_{p}{ }^{*}$.

Proof. Define $f(x)=F^{-1}[[x] / p+F(x-[x]) / p], 0 \leqq x \leqq p$. Clearly, $f(x) \in E_{p}$, and since $F(x)$ satisfies the functional equation (B), it must be the associated function for $f(x)$. This, in turn, implies that $f(x)$ $\in E_{p}{ }^{*}$. Since any function in $E_{p}{ }^{*}$ which has $F(x)$ as its associated function must satisfy the above equation, $f(x)$ is unique.

3.1. Theorems 1 and 2 completely characterize those one-one correspondences between real numbers and sequences of integers $\bmod p$ which can be obtained by applying the algorithm (A) with 
functions from the class $E_{p}^{*}$. They are simply the correspondences which can be obtained from the class of all continuous, strictly increasing functions $F(x)$ on $[0,1]$ to $[0,1]$ by defining the sequence corresponding to the number $\gamma$ to be the sequence of integers in the non- $(p-1)$-terminating expansion of $F(\gamma)$ as a decimal to the base $p$.

3.2. Also as a result of Theorems 1 and 2 we can state that $a$ necessary and sufficient condition for a function $f(x)$ to belong to the class $E_{p}^{*}$ is that it have the form $f(x)=F^{-1}[[x] / p+F(x-[x]) / p], 0 \leqq x \leqq p$, where $F(x)$ is a continuous, strictly increasing function on $[0,1]$ to $[0,1]$.

As an example, if $p=2$ and $F(x)=x^{n}$, then

$$
f(x)= \begin{cases}x / 2^{1 / n}, & 0 \leqq x \leqq 1 \\ {\left[(x-1)^{n}+1\right]^{1 / n} / 2^{1 / n},} & 1 \leqq x \leqq 2 .\end{cases}
$$

From this we can give a periodic representation of the $n$th root of a rational number $R<1$. In fact, $R^{1 / n}=f\left(r_{1}+f\left(r_{2}+\cdots\right)\right)$, where $0 . r_{1} r_{2} \ldots$ is the dyadic expansion of $R$.

3.3. Let $A$ be any denumerable dense set on $[0,1]$ with $0 \in A$ and $1 \in A$ (for example, the set of all algebraic numbers on $[0,1]$ ). There exists a function $f(x) \in E_{p}^{*}$ such that a number $\alpha$ is in $A$ if and only if $\alpha=f\left(a_{1}+f\left(a_{2}+\cdots+f\left(a_{n}\right) \cdots\right)\right)$ for some finite sequence of integers $a_{1}, a_{2}, \cdots, a_{n}$ with $0 \leqq a_{j} \leqq p-1$. For, by virtue of Theorem 2 , it is sufficient to show that there exists a continuous, strictly increasing function $F(x)$ on $[0,1]$ to $[0,1]$ which maps the set $A$ onto the entire set $T$ of numbers on $[0,1]$ whose decimal expansions to the base $p$ are terminating.

To define the function $F(x)$, order the sets $A: a_{0}=0, a_{1}=1, a_{2}, \cdots$, and $T: t_{0}=0, t_{1}=1, t_{2}, \cdots$. Define $F(0)=0, F(1)=1$, and $F\left(a_{2}\right)=t_{2}$. Now suppose that $F(x)$ has been defined in monotonically increasing fashion at all points $a_{j}, j<n$, with values in the set $T$. Let $a_{i}$ be the largest number $a_{j}$ such that $j<n$ and $a_{j}<a_{n}$, and let $a_{k}$ be the smallest number $a_{j}$ such that $j<n$ and $a_{j}>a_{n}$. Then define $F\left(a_{n}\right)$ to be the first number $t$ in $T$ such that $F\left(a_{i}\right)<t<F\left(a_{k}\right)$. Clearly, $F(x)$ is thus defined on the set $A$ and is strictly increasing. Moreover, on $A, F(x)$ takes on every value in the set $T$. Since both $T$ and $A$ are dense in $[0,1]$, we can extend $F(x)$ continuously to the entire interval $[0,1]$ and obtain the desired function.

4.0. The many-one case. Let us turn now to the problem of characterizing those many-one correspondences between real numbers and sequences of integers mod $p$ which can be obtained by applying the algorithm (A) with functions from the class $E_{p}-E_{p}^{*}$. In terms of 
associated functions, the problem is that of characterizing the functions $F(x)$ which are associated with functions $f(x)$ in $E_{p}-E_{p}^{*}$.

4.1. A continuous, nondecreasing function $F(x)$ on $[0,1]$ with $F(0)=0$ and $F(1)=1$ will be said to have the property $(\mathrm{P})$ if, whenever a number $0 . a_{1} a_{2} \cdots$ (decimal to the base $p$ ) is a level value for $F(x)$, each of the numbers $0 . a_{2} a_{3} \cdots$ and $0 . i a_{1} a_{2} \cdots, i=0,1, \cdots$, $p-1$, is also a level value for $F(x)$. It shall be understood that if a number which can be represented by both a terminating and a $(p-1)$-terminating decimal is a level value, then every such number between 0 and 1 , and one, but not necessarily both, of the numbers 0 and 1 , must also be level values.

If $G(x)$ is defined for $0 \leqq x \leqq p$ by $G(x)=[x] / p+F(x-[x]) / p$, and if $F(x)$ has the property (P), then a number $t$ is a level value for $F(x)$ if and only if it is a level value for $G(x)$. For suppose s $^{2} t=0 . a_{1} a_{2} \ldots$ is a level value for $F(x)$; then property $(\mathrm{P})$ implies that $F\left(x_{1}\right)=F\left(x_{2}\right)$ $=t p-a_{1}=0 . a_{2} a_{3} \cdots$ for $0<x_{1}<x_{2}<1$. Consequently, $G\left(x_{1}+a_{1}\right)$ $=a_{1} / p+F\left(x_{1}\right) / p=a_{1} / p+F\left(x_{2}\right) / p=G\left(x_{2}+a_{1}\right)=t$, so $t$ is a level value for $G(x)$. Conversely, if $G\left(\xi_{1}\right)=G\left(\xi_{2}\right)=t$ for $\xi_{1} \neq \xi_{2}$ and $\left[\xi_{1}\right]=\left[\xi_{2}\right]$, then $F\left(\xi_{1}-\left[\xi_{1}\right]\right)=F\left(\xi_{2}-\left[\xi_{2}\right]\right)=p t-\left[\xi_{1}\right]=0 . c_{1} c_{2} \cdots$ and property $(\mathrm{P})$ implies that $t=0$. [ $\left.\xi_{1}\right] c_{1} c_{2} \cdots$ is a level value for $F(x)$.

4.2. In $\$ 2.3$ we saw that the associated function of every function in the class $E_{p}-E_{p}^{*}$ is nondecreasing but not strictly increasing on $[0,1]$ to $[0,1]$ and has the property $(P)$. We shall now show that the converse is also true.

THEOREM 3. Let $F(x)$ be a nondecreasing function on $[0,1]$ with $F(0)=0$ and $F(1)=1$. If $F(x)$ is not strictly increasing and has the property $(\mathrm{P})$, then it is the associated function for infinitely many functions $f(x)$ in the class $E_{p}-E_{p}^{*}$.

Proof. As in $\$ 4.1$, define $G(x)=[x] / p+F(x-[x]) / p$ for $0 \leqq x \leqq p ;$ then we seek a function $f(x)$ in $E_{p}$ such that $F[f(x)]=G(x)$.

For every number $t, 0 \leqq t \leqq 1$, denote by $E_{F}(t)$ the set (either an interval or a single point) of points $x$ such that $F(x)=t$. Define $m_{F}(t)=$ g.l.b. $E_{F}(t)$ and $M_{F}(t)=$ l.u.b. $E_{F}(t)$. Similarly, denote by $E_{G}(t)$ the set of points $x$ such that $G(x)=t$, and define $m_{G}(t)=$ g.l.b. $E_{G}(t)$ and $M_{G}(t)=$ l.u.b. $E_{G}(t)$. Now define $f\left[m_{G}(t)\right]=m_{F}(t)$ and $f\left[M_{G}(t)\right]=M_{F}(t)$. Then $f(x)$ is defined for every point on $[0, p]$ not in the interior of an interval of constancy of $G(x)$. The fact that $F(x)$ and $G(x)$ are nondecreasing and that $t$ is a level value for $F(x)$ if and only if it is a level value for $G(x)$ insures that $f(x)$ is strictly increasing.

${ }^{2}$ If $t=q / p$ we take the terminating or nonterminating decimal representation for $t$ according as 0 or 1 is a level value for $F(x)$. 
On every open interval of constancy of $G(x)$, that is, on the intervals $m_{G}(t)<x<M_{G}(t)$, we can define $f(x)$ quite arbitrarily subject only to the restrictions that it must be strictly increasing and must take on every value between $m_{P}(t)$ and $M_{F}(t)$. The function $f(x)$ is then defined on the entire interval $[0, p]$, is strictly increasing, and takes on every value in the interval $[0,1]$. Consequently, it is continuous and belongs to the class $E_{p}$. Moreover, it follows at once from the definition of $f(x)$ that $F[f(x)]=G(x)$.

Theorem 3 completes the characterization of those functions $F(x)$ which are associated with functions $f(x)$ in the class $E_{p}-E_{p}^{*}$.

4.3. All of the examples of functions in the class $E_{p}-E_{p}^{*}$ which Everett constructed give rise to correspondences with the following two properties: (1) the only sequences of integers which correspond to more than one real number are terminally periodic; and (2) the set $U$ of those numbers which are associated uniquely with a sequence of integers has measure zero. This led him to raise the following two questions: (1) do there exist functions which in the algorithm (A) associate with more than one real number the same nonterminally periodic sequence of integers? (2) do functions exist with sets $U$ of every measure between 0 and 1 ?

Stated in terms of associated functions the questions are: (1) do there exist functions whose associated functions have nonterminally periodic decimals as level values? (2) do functions exist whose associated functions have their sets of points of increase of every measure between 0 and 1 ? As a result of Theorem 3, we can clearly give an affirmative answer to both of these questions.

5.0. A homeomorphism from $E_{p}$ onto $E_{p}^{*}$. If $f(x)$ is any function in $E_{p}$, then $F^{*}(x)=f(p x)$ is a continuous, strictly increasing function on $[0,1]$ to $[0,1]$. As such it is the associated function for some function $f^{*}(x) \in E_{p}^{*}$. We can thus define a one-one transformation $T$ of $E_{p}$ onto $E_{p}^{*}$ by simply setting $T f=f^{*}$. If $E_{p}$ and $E_{p}^{*}$ are regarded as subsets of the metric space of continuous functions on $[0, p]$ (where the distance between two functions $f$ and $g$ is given by max $|f-g|$ ), this transformation is, in fact, a homeomorphism as will be established in Theorems 4 and 5 .

5.1. In the following sections we shall have occasion to use the theorem ${ }^{3}$ from the theory of functions of a real variable which states that if the functions $f_{1}(x), f_{2}(x), \cdots$ are continuous on the closed interval $[a, b]$, then a necessary and sufficient condition that $\lim _{n \rightarrow \infty} f_{n}(x)$ $=f_{0}(x)$ uniformly on $[a, b]$ is that $\lim _{n \rightarrow \infty}^{x \rightarrow x_{0}} f_{n}(x)=f_{0}\left(x_{0}\right)$ for each point $x_{0}$

See, for example, Townsend, Functions of real variables, p. 350. 
in $[a, b]$. As an easy application of this theorem we have the fact that if $f_{1}(x), f_{2}(x), \cdots$ is any sequence of continuous, nondecreasing functions on $[a, b]$ which converges at every point of $[a, b]$ to a continuous function $f_{0}(x)$, then $f_{0}(x)$ is nondecreasing and the convergence is uniform on $[a, b]$.

5.2. TheOREM 4. If the functions $f_{n}(x) \in E_{p}, n=1,2, \cdots$, converge on $[0, p]$ to the function $f_{0}(x) \in E_{p}$, then their corresponding associated functions $F_{n}(x), n=1,2, \cdots$, converge uniformly on $[0,1]$ to $F_{0}(x)$, the associated function for $f_{0}(x)$.

Proof. It follows from $\$ 5.1$ that it will be sufficient to show that $\lim _{n \rightarrow \infty} F_{n}(x)=F_{0}(x)$ pointwise on $[0,1]$. Moreover, if we show that $\lim _{n \rightarrow \infty} F_{n}(x)=F_{0}(x)$ at each point $x$ on the open interval $(0,1)$ for which $0<F_{0}(x)<1$, then the convergence on the entire interval $[0,1]$ follows from the fact that the functions $F_{n}(x)$ are continuous, nondecreasing with $F_{n}(0)=0$ and $F_{n}(1)=1, n=0,1, \ldots$.

Let $\gamma$ be any point on $(0,1)$ such that $0<F_{0}(\gamma)<1$, and let $\epsilon$ be an arbitrary positive number. Choose two numbers $A=0 . a_{1} a_{2} \cdots a_{k}$ $<F_{0}(\gamma)$ and $B=0 . b_{1} b_{2} \cdots b_{l}>F_{0}(\gamma)$ such that $F_{0}(\gamma)-A<\epsilon$ and $B-F_{0}(\gamma)<\epsilon$. Then

$f_{0}\left(a_{1}+\cdots+f_{0}\left(a_{k}\right) \cdots\right)=\alpha<\gamma<\beta=f_{0}\left(b_{1}+\cdots+f_{0}\left(b_{l}\right) \cdots\right)$.

$\$ 5.1$ implies that $\lim _{n \rightarrow \infty} f_{n}(x)=f_{0}(x)$ uniformly on $[0, p]$, and hence, for $n$ sufficiently large,

$$
\begin{aligned}
f_{n}\left(a_{1}+\cdots+f_{n}\left(a_{k}\right)\right. & \cdots) \\
& =\alpha_{n}<\gamma<\beta_{n}=f_{n}\left(b_{1}+\cdots+f_{n}\left(b_{l}\right) \cdots\right) .
\end{aligned}
$$

Moreover, $F_{n}\left(\alpha_{n}\right)=F_{0}(\alpha)=A$ and $F_{n}\left(\beta_{n}\right)=F_{0}(\beta)=B$. From the monotonicity of the functions $F_{n}(x)$ it follows that for $n$ sufficiently large, $A \leqq F_{n}(\gamma) \leqq B$ and hence $\left|F_{0}(\gamma)-F_{n}(\gamma)\right|<\epsilon$.

As a corollary to Theorem 4 we have the continuity of the transformation $T^{-1}$ from $E_{p}^{*}$ onto $E_{p}$.

5.3. Theorem 5. Let $F_{0}(x), F_{1}(x), \cdots$ be a sequence of continuous, strictly increasing functions on $[0,1]$ to $[0,1]$, and let $f_{0}(x)$, $f_{1}(x), \cdots$ be the sequence of functions in the class $E_{p}^{*}$ such that $F_{n}(x)$ is the associated function for $f_{n}(x), n=0,1, \ldots$ If $\lim _{n \rightarrow \infty} F_{n}(x)$ $=F_{0}(x)$ on $[0,1]$, then $\lim _{n \rightarrow \infty} f_{n}(x)=f_{0}(x)$ uniformly on $[0, p]$.

PROOF. Since each of the functions $F_{n}(x)$ is strictly increasing, $n=0,1, \cdots$, the inverse functions $F_{1}^{-1}(t), F_{2}^{-1}(t), \cdots$ converge to $F_{0}^{-1}(t)$, and by $\S 5.1$ the convergence is uniform. Moreover, it will again 
be sufficient to establish that $\lim _{n \rightarrow \infty} f_{n}(x)=f_{0}(x)$ at every point $x$ on $[0, p]$. The theorem follows immediately then from $\$ 5.1$ and the fact that

$$
f_{n}(x)=F_{n}^{-1}\left[[x] / p+F_{n}(x-[x]) / p\right], 0 \leqq x \leqq p, n=0,1, \cdots
$$

Theorem 5 establishes the continuity of $T$ from $E_{p}$ onto $E_{p}^{*}$.

The Ohio State University

\section{THE NONLINEAR DIFFERENTIAL EQUATION$$
y^{\prime \prime}+p(x) y+c y^{-3}=0
$$ \\ EDMUND PINNEY ${ }^{1}$}

Among the limited number of nonlinear differential equations whose exact solutions are known is to be included

$$
y^{\prime \prime}(x)+p(x) y(x)+c / y^{3}(x)=0,
$$

for $c$ constant and $p(x)$ given. The general solution for which $y\left(x_{0}\right)$ $=y_{0} \neq 0, y^{\prime}\left(x_{0}\right)=y_{0}^{\prime}$ is

$$
y(x)=\left[u^{2}(x)-c W^{-2} v^{2}(x)\right]^{1 / 2},
$$

where $u, v$ form a fundamental set of solutions of the linear equation

$$
y^{\prime \prime}(x)+p(x) y(x)=0
$$

for which $u\left(x_{0}\right)=y_{0}, u^{\prime}\left(x_{0}\right)=y_{0}^{\prime}, v\left(x_{0}\right)=0, v^{\prime}\left(x_{0}\right) \neq 0$, where $W$ is their Wronskian: $W=u v^{\prime}-u^{\prime} v=$ const. $\neq 0$, and where the radical in (2) stands for that root which at $x_{0}$ has the value $y_{0}$.

The proof is very simple and will be omitted.

University of California, Berkeley

Received by the editors July 18, 1949.

1 Done in connection with the Office of Naval Research contract N6 ONR 251 T.O. 2 at Stanford University. I am indebted to Prof. R. E. Langer for a simplification of the result. 